\title{
VALIDACIÓN DEL NURSING ACTIVITIES SCORE EN UNIDADES DE CUIDADOS INTENSIVOS CHILENAS
}

\section{VALIDATION OF THE NURSING ACTIVITIES SCORE IN CHILEAN INTENSIVE CARE UNITS ${ }^{1}$}

\author{
Maria Cecilia Toffoletto* \\ Katiuska Lidice Reynaldos Grandón ** \\ Yerko Molina Muñoz ${ }^{* * *}$ \\ Katia Grillo Padilha ${ }^{* * * *}$
}

\begin{abstract}
RESUMEN
Objetivo: Analizar el grado de preservación de las propiedades psicométricas del Nursing Activities Score (NAS), según análisis de confiabilidad y validez del instrumento. Material y método: Estudio instrumental para la adaptación y validación de un instrumento que mide la carga de trabajo de enfermería en Unidades de Cuidados Intensivos y que abarca más del 80\% de las actividades de enfermería, estimando la asignación diaria de pacientes al personal de enfermería, según las necesidades de cuidados de los mismos. La validación se realizó en seis unidades de cuidados intensivos de dos hospitales de Santiago de Chile. Resultados: La media NAS fue de 70,86\%. Para el análisis factorial exploratorio se excluyeron los ítems que no presentaron variabilidad (2, 3, 15, 16 y 18). Se utilizó el método de extracción de factores de mínimos cuadrados en el programa Factor y se decidió la solución de 1 factor que explicó el 12,8 \% de la varianza total del instrumento. La estructura factorial propuesta agrupó a los ítems en 1 factor y los ítems 1, 5, 6, 7, 8, 13, 14, 19, 20, 22 y 23 no presentaron una comunalidad adecuada. El estadístico de Kuder-Richardson fue de 0,886. La validez de criterio externo presentó una correlación alta y positiva $(\mathrm{r}=0,601)$. Conclusión: Debido a la relevancia teórica y práctica y atingencia conceptual de los ítems reportada en el proceso de validación de contenido, se ha decidido no recomendar la eliminación de estos ítems y seguir haciendo estudios de validación en otras poblaciones.
\end{abstract}

Palabras clave: Carga de Trabajo; Enfermería; Estudios de Validación; Unidades de Cuidados Intensivos.

\section{ABSTRACT}

Objective: Analyse the degree to which psychometric properties are preserved in the Nursing Activities Score through assessments of instrument reliability and validity. Method: Assessments of instrument adaptation and validation that measures the workload of nursing in Intensive Care Units and that covers more than $80 \%$ of nursing activities, estimating the daily allocation of patients to the nursing staff according to the patients' care needs. The instrument's reliability was assessed in six intensive care units located in two hospitals in Santiago,

\footnotetext{
${ }^{1}$ Fuente apoyo financiero. Universidad Andrés Bello Proyecto regular interno DI-726-15/CB

* Enfermera. Doctor en Enfermería. Profesor asociado de la Facultad de Enfermería Universidad Andrés Bello. Santiago, Chile.Email: mtoffoletto@inacap.cl

${ }^{*}$ Enfermera-matrona. Doctor en Ciencias Empresariales. Profesor asociado de la Facultad de Enfermería Universidad Andrés Bello. Santiago, Chile. Email: katiuska.reynaldos@unab.cl. Autor de correspondencia.

${ }_{* * *}^{*}$ Psicólogo. Máster en psicología de la salud y epidemiología. Profesor de la Facultad de Psicología Universidad Adolfo Ibáñez. Santiago, Chile. Email: yerko.molina@uai.cl

${ }^{* * * *}$ Enfermera. Doctor en Enfermería. Profesor titular de la Escola de Enfermagem Universidade de São Paulo. São Paulo, Brasil.Email: kgpadilh@usp.br
} 
Chile. Results: The mean nursing activities score was $70.86 \%$. For exploratory factor analysis, items without variability were excluded $(2,3,15,16$ and 18$)$. The least squares factor extraction method was used, executed in the Factor software, and it resulted in one factor explaining $12.8 \%$ of total instrument variance. The proposed factor structure grouped items in one factor; however, items 1, 5, 6, 7, 8, 13, 14, 19, 20, 22 and 23 did not present adequate communality. The calculated value for the Kuder-Richardson formula was 0.886 . The Nursing Activities Score instrument was highly and positively correlated with the Therapeutic Intervention Scoring System ( $\mathrm{r}$ $=0.601)$, used as an external validation criterion. Conclusion: Due to theoretical and practical relevancy, as well as conceptual connections of the items selected during the content validation process, we recommend that these items are not eliminated from the Nursing Activities Score instrument. Additional validation studies should be conducted in other populations.

Key words: Workload; Nursing; Validation Studies; Intensive Care Units.

Fecha recepción: 28/09/17 Fecha aceptación: 25/06/18

\section{INTRODUCCIÓN}

El cuidado al paciente grave exige la utilización de alta tecnología, diversas y complejas intervenciones terapéuticas, además del soporte constante a las familias, el cual diferencia las unidades de cuidados intensivos (UCI) de los demás servicios en cuanto a la demanda de los recursos humanos en enfermería ${ }^{(1)}$. La inadecuada dotación de personal de enfermería en las UCI y consecuente aumento de la carga de trabajo, debilita la calidad de la asistencia prestada al paciente e incide directamente en los indicadores asistenciales relacionados a las tasas de infección, errores de medicación, caídas, entre otros, llevando a un aumento del tiempo de hospitalización y de cos$\operatorname{tos}^{(2)}$.

La carga de trabajo de enfermería es definida como todas las actividades de cuidado en un determinado período y el tiempo utilizado para la ejecución de las mismas ${ }^{(3)}$. Entre los diversos instrumentos que miden la carga de trabajo de enfermería en las UCI, el instrumento Nursing Activities Score (NAS) tiene la ventaja de abarcar más del 80\% de las actividades de cuidados, estimando la asignación diaria de pacientes al personal de enfermería, de acuerdo a las necesidades de cuidados de los mismos ${ }^{(4)}$.

Originario del Simplified Therapeutic Inter- vention Scoring System (TISS-28), el NAS fue desarrollado el año 2003 y está constituido por 23 actividades relacionadas a cuidados de monitoreo y control, soporte ventilatorio, soporte cardiovascular, soporte renal, soporte neurológico, soporte metabólico, intervenciones específicas, además de soporte a los familiares y tareas de gestión. En el instrumento, las actividades son registradas diariamente por los enfermeros, en base a los cuidados prestados en las últimas 24 horas y cada una de las 23 actividades posee un puntaje. La suma de los puntos representa, en porcentaje, el gasto del tiempo que el paciente necesitó del $100 \%$ del tiempo de trabajo de enfermería ${ }^{(5)}$.

Estudios evidencian la efectividad de este instrumento en la medida de la carga de trabajo en UCI. Un estudio brasileño, realizado en una unidad neonatal, analizó la carga de trabajo de enfermería por medio del NAS y calculó la cantidad ideal del personal de enfermería, comparándolo con el actual. Los hallazgos demostraron una brecha importante entre el personal existente y el que realmente se necesitaba, según la elevada demanda de trabajo a la cual estaba sometido diariamente $^{(6)}$.

Otro estudio realizado en 19 UCIs de siete países, describió la carga de trabajo de enfermería según el NAS. Los resultados arrojaron una puntuación media total de $72,81 \%$ con valores de 44,46\% en España, 101,8\% en Noruega, 83,0\% 
en Polonia, 64,59\% en Grecia y 57,1\%, 54,0\% y $51,0 \%$ en Egipto, Brasil y Países Bajos, respectivamente, cifras consideradas elevadas porque muestran que los pacientes necesitan más de $50 \%$ del tiempo del personal de enfermería ${ }^{(7)}$. Otro estudio en Noruega, con 235 pacientes de UCIs generales, también evidenció un puntaje elevado, cuya media NAS fue de $96,24 \%{ }^{(8)}$.

La evidencia también ha verificado la efectividad del NAS en la medida de la carga de trabajo de enfermería de pacientes con distintos diagnósticos y momentos de hospitalización. Un estudio realizado en Madrid, cuyo objetivo fue analizar la carga laboral en los momentos de ingreso y alta de tres grupos de pacientes con diagnósticos distintos, concluyó que el grupo que presentó menor carga de trabajo al ingreso presentó a lo largo de su hospitalización el mismo puntaje de pacientes que ingresaron en condiciones más críticas. Frente a los resultados, los autores resaltaron la importancia de los hallazgos al tener en cuenta la planificación de los recursos humanos ${ }^{(9)}$.

En Chile, la distribución diaria del personal de enfermería en Unidades de Cuidados Intensivos (UCI) sigue lo que indican las guías 2004, referidas a la organización y funcionamiento de estas unidades $^{(10)}$, en donde se establece que la dotación de profesionales de enfermería en (UCI) es de uno para tres pacientes y en Unidades de Tratamiento Intensivo (UTI) es de uno para seis pacientes. No se han encontrado estudios recientes que analicen esta relación en nuestro país.

De lo anterior, y con el interés de disponer de un instrumento validado en Chile que mida la carga de trabajo de enfermería desde la mirada de las necesidades de cuidados de los pacientes, que tenga reconocimiento internacional y que sea valioso para la gestión de recursos humanos de enfermería, esta investigación analizó el grado de preservación de las propiedades psicométricas del Nursing Activities Score, según análisis de la confiabilidad y validez, aplicado a una muestra de pacientes hospitalizados en UCI de dos hospitales de Santiago de Chile.

\section{MATERIAL Y MÉTODO}

Tipo de estudio: Consistió en un estudio instrumental para la validación de instrumento conformado por las siguientes etapas: Evaluar la validez de constructo, evaluar la validez de criterio y determinar la consistencia interna ${ }^{(11-13)}$.

Población y muestra: Los datos fueron recolectados entre junio y noviembre de 2016, en seis Unidades de Cuidados Intensivos de dos hospitales de Santiago de Chile. El número de pacientes establecidos para la conformación de la muestra fue designado de acuerdo a las recomendaciones de Martínez-Arias ${ }^{(14)}$. La muestra por conveniencia fue conformada por 126 pacientes, de un universo total de 264 pacientes, que cumplieron los criterios de inclusión: Mayores de 16 años y con permanencia mínima de 24 h de hospitalización en la UCI. Las variables de caracterización de la muestra fueron 3: sexo (masculino, femenino); procedencia (Urgencia, Unidad de Cuidados Intensivos, Unidad de tratamiento Intermedio, Pabellón, Unidad de hospitalización, Otro hospital) y Tipo de tratamiento (Clínico, Clínico-quirúrgico, Quirúrgico).

Instrumentos recolectores de datos: Un factor limitante de la utilización del NAS original es su idioma inglés, razón por la cual en América Latina se encuentra adaptado y validado en Brasil ${ }^{(1)}$ y adaptado en Colombia ${ }^{(15)}$. Así, para este estudio se realizó traducción y adaptación al español hablado en Chile del NAS y fue utilizado el TISS-28 para el análisis de criterio externo.

El instrumento NAS está constituido por siete grandes categorías que contemplan 23 actividades: actividades básicas (8 actividades), soporte ventilatorio (3 actividades), soporte cardiovascular (4 actividades), soporte renal (2 actividades), soporte neurológico (1 actividad), soporte metabólico (3 actividades) e intervenciones específicas (2 actividades). En el instrumento, las actividades son registradas por los enfermeros en base a los cuidados prestados en las últimas $24 \mathrm{~h}$ 
y cada una de las 23 actividades posee un puntaje. La suma de los puntos representa en porcentaje el gasto del tiempo que el paciente necesitó del $100 \%$ del tiempo de trabajo de enfermería ${ }^{(5)}$. Para su adaptación con equivalencia semántica e idiomática las etapas metodológicas fueron: la traducción y retrotraducción, la evaluación de la validez de contenido y la adaptación lingüística a la población a evaluar. Posterior a la traducción y retrotraducción, dos investigadores y un colaborador externo realizaron una síntesis a partir de las versiones anteriores, es decir, del instrumento original en inglés, las traducciones y retrotraducciones hasta llegar a un primer informe en el idioma español. La primera versión fue sometida a la evaluación de contenido por cinco jueces expertos y se utilizó el coeficiente de validez de contenido. La validez de contenido y el índice de $\operatorname{Lyn}^{(13)}$ fueron entre 0,6 y 1,0 y entre 80 y 100 , respectivamente. Para la adaptación lingüística se conformó un grupo focal con ocho enfermeros clínicos que evaluaron la comprensión y viabilidad del instrumento. Como resultado, algunas expresiones fueron ajustadas a la cultura chilena, que obligó a generar una segunda versión del instrumento. En la prueba piloto, el tiempo promedio de aplicación de esta nueva versión fue de 10,2 min, siendo considerado comprensible y viable en su aplicación pues no hubo alteraciones en el contenido y se consideró, esta segunda versión del NAS, como la definitiva y final.

El instrumento TISS-28 está conformado por siete categorías: actividades básicas, soporte ventilatorio, soporte renal, soporte neurológico, soporte metabólico e intervenciones específicas. Cada categoría está constituida por ítems específicos con un puntaje que varía de uno a ocho. Para cada punto se estima 10,6 min del tiempo de trabajo de enfermería ${ }^{(16)}$. Procedimiento: La aplicación de los instrumentos a la muestra fue realizada en un período de 6 meses. Previo a ello, se realizaron dos reuniones con todos los enfermeros participantes de las UCI de cada hospital, los cuales recibieron informaciones respecto a los objetivos, procedimientos del estudio, instrumento y su aplicación. La participación de los enfermeros en la aplicación del instrumento fue voluntaria y anónima, posterior a la firma del Consentimiento Informado por los mismos. Participaron en el estudio 29 enfermeros, quienes aplicaron el NAS diariamente a todos los pacientes hospitalizados en las UCI que cumplían con los criterios de inclusión. Es importante destacar que la aplicación del instrumento fue realizada solo una vez por paciente y fue supervisada por dos investigadores con experiencia en pacientes críticos.

Análisis de datos: Para evaluar la validez de constructo del instrumento se utilizó un análisis factorial exploratorio con el método de extracción de factores de mínimos cuadrados en el programa FACTOR, que permite el análisis de matrices policóricas con rotación oblicua oblimin directo. Cabe señalar que el Análisis Factorial Confirmatorio no fue realizado por no haber una fuerte fundamentación empírica y conceptual para poder guiar la especificación y evaluación del modelo factorial ${ }^{(17)}$. Para el análisis de confiabilidad se utilizó el estadístico de Kuder-Richardson. Para evaluar congruencia entre NAS y el criterio externo TISS-28, se utilizó análisis de correlación de Pearson.

Resguardos éticos: El proyecto fue certificado por el Comité de Ética de la Facultad de Enfermería de la Universidad Andrés Bello con el registro L1/ CECENF/81.

\section{RESULTADOS}

Características de la muestra: Los pacientes en su mayoría fueron de sexo masculino (58,73\%), con una media de edad de 53,93 años, siendo el promedio para los hombres de 50,34 años $(\mathrm{DE}=17,08)$ y para las mujeres de 59,57 años $(\mathrm{DE}=19,74)$. En cuanto a la procedencia de los pacientes al ingreso a las UCI y tipo de tratamiento, el servicio de urgencia $(42,86 \%)$ y el tratamiento clínico (50,00\%), respectivamente, fueron los más frecuentes (Tabla 1). La media NAS presentada por la muestra fue de 70,86\% (Tabla 1). 
Tabla 1. Análisis descriptivo de la muestra según sexo, procedencia y tipo de tratamiento.

\begin{tabular}{llcc}
\hline Variables & & Frecuencia & $\%$ \\
\hline \multirow{3}{*}{ Sexo } & Masculino & 74 & 58,73 \\
& Femenino & 52 & 41,27 \\
& Total & 126 & 100 \\
\hline \multirow{3}{*}{ Procedencia } & Urgencia & 54 & 42,86 \\
& Otro hospital & 28 & 22,22 \\
& Unidad de Cuidados Intensivos & 21 & 16,67 \\
& Pabellón & 11 & 8,73 \\
& Unidad de Tratamiento Intermedio & 9 & 7,14 \\
& Unidad de hospitalización & 3 & 2,38 \\
Tipo de tratamiento & Total & 126 & 100 \\
& Clínico & 63 & 50 \\
& Clínico-quirúrgico & 37 & 29,37 \\
& Quirúrgico & 26 & 20,63 \\
\hline
\end{tabular}

Validez de constructo y confiabilidad: Se revisó la adecuación de los reactivos, en especial la variabilidad de los ítems del instrumento. Las frecuencias de los ítems variaron de acuerdo a los dominios del instrumento: Actividades Básicas, Asistencia Respiratoria, Asistencia Cardiovascular, Asistencia Renal, Asistencia Neurológica, Asistencia Metabólica, Intervenciones Específicas. A pesar de que ningún ítem presentó una frecuencia de 100\%, algunos sí presentaron frecuencias elevadas, a saber: el ítem 2 relativo a "control de exámenes de laboratorio, bioquímica y microbiología" (99,4\%; $\mathrm{n}=125)$; el ítem 3 , referente a "preparación y administración de medicamentos, excluidos los medicamentos vasoactivos" (99,4\%; $n=125)$; el ítem 15 , referente a "resucitación cardiopulmonar después de un paro durante las últimas 24 h" $(97,6 \% ; n=123)$; el ítem 16, referente a "técnicas de hemofiltración, técnicas de diálisis” (96,0\%; $n=121)$ y por último el ítem 18, referente a "Medición de presión intracraneal $(95,2 \% ; n=120)$ (Tabla 2).

Tabla 2. Distribución de los ítems que presentaron frecuencias elevadas según la necesidad de cuidado de los pacientes.

\begin{tabular}{|c|c|c|c|c|c|}
\hline \multirow[t]{2}{*}{$\mathrm{N}^{\circ}$} & \multirow[t]{2}{*}{ Ítem } & \multicolumn{2}{|c|}{ Si } & \multicolumn{2}{|c|}{ No } \\
\hline & & $\mathrm{n}$ & $\%$ & $\mathrm{n}$ & $\%$ \\
\hline la & $\begin{array}{l}\text { Control de los signos vitales por horario, registro y cálculo constante del balance hí- } \\
\text { drico. }\end{array}$ & 42 & 33,3 & 84 & 66,7 \\
\hline $1 b$ & Presente a pie de cama y observación continua o activa durante 2 h o más. & 44 & 34,9 & 82 & 65,1 \\
\hline $1 c$ & Presente a pie de cama y observación activa durante 4 h o más. & 41 & 32,5 & 85 & 67,5 \\
\hline 2 & Control de exámenes de laboratorio, bioquímica y microbiología. & 125 & 99,4 & 1 & 0,8 \\
\hline 3 & $\begin{array}{l}\text { Preparación y administración de medicamentos, excluidos los medicamentos vasoac- } \\
\text { tivos. }\end{array}$ & 125 & 99,4 & 1 & 0,8 \\
\hline $4 \mathrm{a}$ & Ejecución de procedimientos de higiene. & 55 & 43,7 & 71 & 56,3 \\
\hline $4 \mathrm{~b}$ & Ejecución de procedimientos de higiene durante $2 \mathrm{~h}$ o más en cualquier turno. & 49 & 38,9 & 77 & 61,1 \\
\hline $4 \mathrm{c}$ & Ejecución de procedimientos de higiene durante 4 h o más en cualquier turno. & 23 & 18,3 & 103 & 81,7 \\
\hline
\end{tabular}


Continuación Tabla II.

\begin{tabular}{|c|c|c|c|c|c|}
\hline 5 & Cuidado de drenajes de todo tipo (exceptuando sondas gástricas). & 50 & 39,7 & 76 & 60,3 \\
\hline $6 a$ & Realización de procedimiento(s) hasta tres veces en $24 \mathrm{~h}$. & 42 & 33,3 & 84 & 66,7 \\
\hline 6 & $\begin{array}{l}\text { Realización de procedimiento(s) con una frecuencia mayor que } 3 \text { veces en } 24 \text { h, o con } \\
\text { dos enfermeras, cualquier frecuencia. }\end{array}$ & 61 & 48,4 & 65 & 51,6 \\
\hline $6 c$ & Realización de procedimiento con tres o más enfermeras, cualquier frecuencia. & 23 & 18,3 & 103 & 81,7 \\
\hline $7 \mathrm{a}$ & Apoyo y cuidado de los familiares o del paciente. & 115 & 91,3 & 11 & 8,7 \\
\hline f & $\begin{array}{l}\text { Apoyo y cuidado de los familiares o del paciente que requieran una completa dedica- } \\
\text { ción por } 3 \text { h o más. }\end{array}$ & 10 & 7,9 & 116 & 92,1 \\
\hline 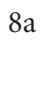 & $\begin{array}{l}\text { Ejecutar labores de rutina, como el procesamiento de datos clínicos, solicitud de exáme- } \\
\text { nes, intercambio de información profesional (por ejemplo, rondas de visitas médicas). }\end{array}$ & 88 & 69,8 & 38 & 30,2 \\
\hline b & $\begin{array}{l}\text { Realización de tareas administrativas y de gestión que requieren dedicación completa } \\
\text { durante aproximadamente } 2 \text { h en cualquier turno tales como actividades de investiga- } \\
\text { ción, protocolos en uso, procedimientos de admisión y alta de pacientes. }\end{array}$ & 35 & 27,8 & 91 & 72,2 \\
\hline $8 \mathrm{c}$ & $\begin{array}{l}\text { Realización de tareas administrativas y de gestión que requieren dedicación completa } \\
\text { durante aproximadamente } 4 \text { h o más. }\end{array}$ & 4 & 3,2 & 122 & 96,8 \\
\hline 9 & Asistencia respiratoria: cualquier forma de ventilación mecánica. & 109 & 86,5 & 17 & 13,5 \\
\hline 10 & Cuidado de vías respiratorias artificiales: tubo endotraqueal o cánula de traqueotomía. & 75 & 59,5 & 51 & 40,5 \\
\hline 11 & Tratamiento para mejorar la función pulmonar. & 11 & 88,1 & 15 & 11,9 \\
\hline 12 & Medicación vasoactiva, independiente del tipo y la dosis. & 41 & 32,5 & 85 & 67,5 \\
\hline 13 & Reposición intravenosa de pérdidas de líquidos. & 27 & 21,4 & 99 & 78,6 \\
\hline 14 & Control del atrio izquierdo. & 23 & 18,3 & 103 & 81,7 \\
\hline 15 & Resucitación cardiopulmonar después de un paro durante las últimas 24 h. & 3 & 2,4 & 123 & 97,6 \\
\hline 16 & Técnicas de hemofiltración, técnicas de diálisis. & 5 & 4 & 121 & 96 \\
\hline 17 & Medición cuantitativa de orina (por ejemplo, mediante sonda vesical). & 110 & 87,3 & 16 & 12,7 \\
\hline 18 & Medición de presión intracraneal. & 6 & 4,8 & 120 & 95,2 \\
\hline 19 & Tratamiento de las complicaciones de acidosis/alcalosis metabólica. & 13 & 10,3 & 113 & 89,7 \\
\hline 20 & Nutrición Parenteral Total Central. & 19 & 15,1 & 107 & 84,9 \\
\hline 21 & Alimentación enteral mediante sonda gástrica u otros medios gastrointestinales. & 77 & 61,1 & 49 & 38,9 \\
\hline 22 & Intervenciones específicas en la unidad de cuidados intensivos. & 65 & 51,6 & 61 & 48,4 \\
\hline 23 & Intervenciones específicas fuera de la unidad de cuidados intensivos. & 44 & 34,9 & 82 & 65,1 \\
\hline
\end{tabular}

Análisis factorial exploratorio: Se excluyeron los ítems 2, 3, 15, 16 y 18, citados anteriormente, por no presentar suficiente variabilidad. Debido a esto, y a que las variables eran dicotómicas u ordinales, se utilizó un análisis factorial sobre matriz policórica con el método de extracción de factores de mínimos cuadrados. Respecto de la adecuación de la matriz al análisis factorial, se utilizó la prueba de esfericidad de Barttlet, cuyo valor $\mathrm{p}$ fue menor a 0,0001 , lo que significó que la matriz policórica no resultó ser una matriz de identidad. Respecto del estadístico KMO, éste tuvo un valor de 0,69984 , lo que significó ser aceptable para el análisis. Para determinar la cantidad de factores ideales a extraer, se analizaron 3 criterios: el criterio de Kaiser, el análisis para- lelo de Horn y el contraste de caída. Respecto a la cantidad de factores, se decidió la solución de 1 factor que explicó el 12,8 \% de la varianza total del instrumento, por considerar el análisis paralelo de Horn el criterio más apropiado de los utilizados y su aproximación al número de factores propuesto por el contraste de caída (Figura 1). Para analizar las cargas factoriales se analizó la correlación de cada uno de los ítems con el factor resultante, usando como criterio una correlación de 0,3 como mínimo para ser considerado dentro del factor. La estructura factorial propuesta agrupó a los ítems en 1 factor, sin embargo, la gran cantidad de ítems que no presentaron una comunalidad adecuada, en conjunto con los ítems que quedaron fuera del análisis por no presentar una 
variabilidad aceptable, no permitieron ser conclusivos respecto del análisis, quedando fuera de la estructura factorial observada los ítems 1, 5, 6, $7,8,13,14,19,20,22$ y 23 . Las comunalidades bajas determinaron que de todos los ítems eva- luados, sólo quedaran en el factor empíricamente observado los ítems $4,9,10,11,12,17$ y 21 , presentando todos los demás ítems correlaciones con el factor bajo 0,3 (Tabla 3 ).

Figura 1. Gráfico de sedimentación.

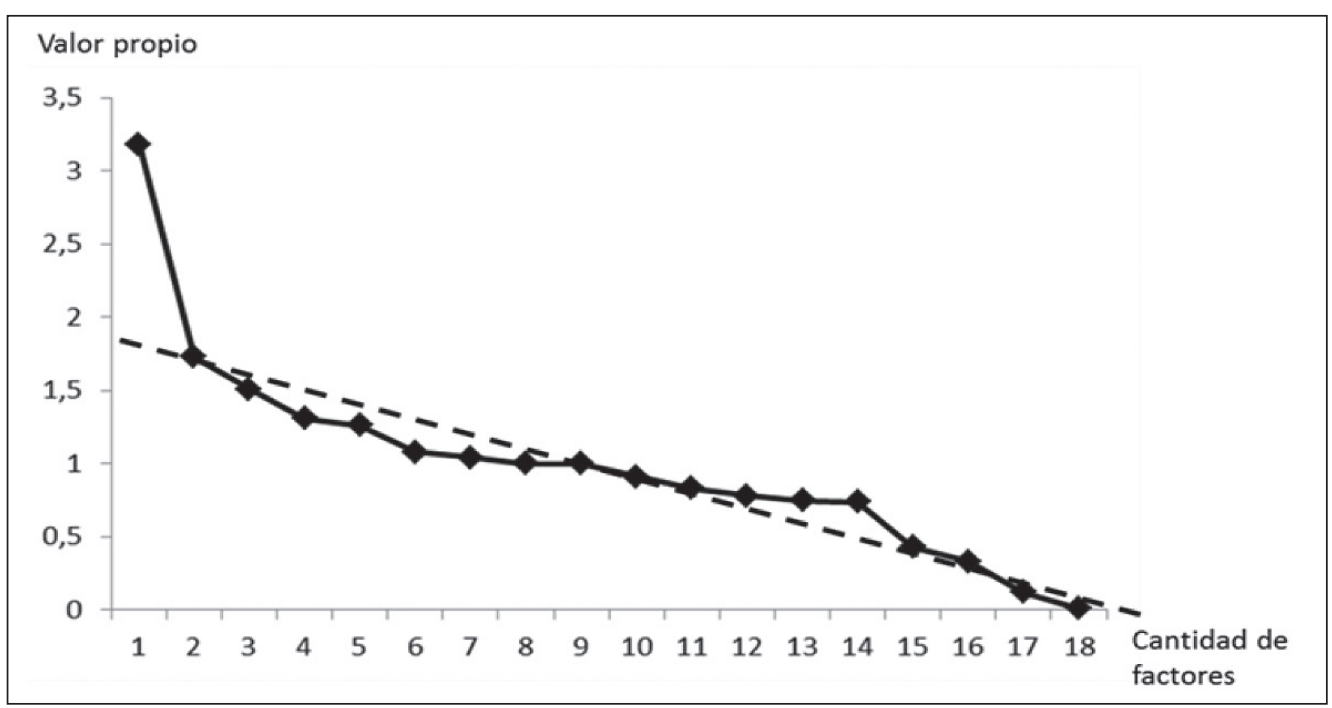

Tabla 3. Cargas factoriales y comunalidades para los ítems del instrumento NAS.

\begin{tabular}{|c|c|c|c|}
\hline $\mathrm{N}^{\circ}$ & Ítem & Carga Factorial & Comunalidad \\
\hline 1 & Control y ajuste. ${ }^{\dagger}$ & 0,223 & 0,050 \\
\hline 4 & Procedimientos de higiene. ${ }^{*}$ & 0,335 & 0,112 \\
\hline 5 & Cuidado de drenajes de todo tipo (exceptuando sondas gástricas)..$^{\dagger}$ & 0,007 & 0,000 \\
\hline 6 & Traslado y cambio de posiciones. ${ }^{\dagger}$ & 0,233 & 0,054 \\
\hline 7 & Apoyo y cuidado de los familiares. ${ }^{\dagger}$ & 0,033 & 0,001 \\
\hline 8 & Labores administrativas y organizacionales. ${ }^{\dagger}$ & 0,028 & 0,001 \\
\hline 9 & Asistencia respiratoria: ventilación mecánica. ${ }^{*}$ & 0,699 & 0,489 \\
\hline 10 & Asistencia respiratoria: cuidado vías respiratorias artificiales. ${ }^{*}$ & 0,859 & 0,737 \\
\hline 11 & Asistencia respiratoria: Tratamientos para mejorar las funciones pulmonares.* & 0,872 & 0,760 \\
\hline 12 & Asistencia cardiovascular: medicamentos vasoactivos.. & 0,367 & 0,135 \\
\hline 13 & Reposición intravenosa de pérdidas de líquidos en altas cantidades. ${ }^{\dagger}$ & 0,060 & 0,004 \\
\hline 14 & $\begin{array}{l}\text { Control del atrio izquierdo: catéter arterial pulmonar, con o sin mediciones del } \\
\text { rendimiento cardiaco. }{ }^{\dagger}\end{array}$ & 0,166 & 0,028 \\
\hline 17 & Asistencia renal: Medidas cuantitativas de orina. ${ }^{*}$ & 0,402 & 0,161 \\
\hline 19 & Tratamientos de las condiciones metabólicas acidosis o alcalosis complicadas. ${ }^{\dagger}$ & 0,069 & 0,005 \\
\hline 20 & Hiperalimentación por vía intravenosa. ${ }^{\dagger}$ & 0,026 & 0,001 \\
\hline 21 & Asistencia metabólica: alimentación enteral mediante sonda gástrica. ${ }^{*}$ & 0,400 & 0,160 \\
\hline 22 & Intervenciones específicas en la unidad de cuidados intensivos. ${ }^{\dagger}$ & 0,035 & 0,001 \\
\hline 23 & $\begin{array}{l}\text { Intervenciones específicas fuera de la unidad de cuidados intensivos: cirugías o } \\
\text { procedimientos de diagnóstico. }\end{array}$ & 0,003 & 0,000 \\
\hline
\end{tabular}

* Ítems incluidos en el análisis factorial; † Ítems excluidos del análisis factorial. 
Confiabilidad: El estadístico de Kuder-Richardson, para el factor encontrado, fue de 0,886 , lo que muestra una alta consistencia interna para los ítems ingresados al factor.

En cuanto a la validez de criterio externo al NAS, se observó entre ambas escalas una correlación alta y positiva $(\mathrm{r}=0,601)$, además de estadísticamente significativa (Sig. (bilateral) $=0,000)$.

\section{DISCUSIÓN}

Los hallazgos, en cuanto a la media de edad de la muestra de 53,93 años, el predominio del sexo masculino, así como la procedencia de los servicios de urgencia y tratamiento clínico, son similares a las características que han tenido los pacientes en estudios internacionales realizados en UCI ${ }^{(18-20)}$.

En cuanto a la media NAS que tuvieron los pacientes del estudio, de 70,86\%, la evidencia es diversa y aunque las puntuaciones entre los países que utilizan el instrumento arrojan medias con alta variabilidad que van desde un $44,46 \%$ a un $101,8 \%$, la puntuación media total de todos ha sido de $72,81 \%$, resultado similar al de este estudio $^{(7)}$.

En cuanto a la variabilidad resultante de los ítems, previo al análisis factorial, también se observa en otros estudios que los ítems relacionados a los exámenes de laboratorio y medicamentos (ítems 2 y 3 ) han presentado frecuencias elevadas. Es el caso de un estudio realizado con pacientes trasplantados ${ }^{(21)}$; otras investigaciones realizadas con pacientes oncológicos en cuidados paliativos ${ }^{(22)}$ y pacientes víctimas de trauma hospitalizados en $\mathrm{UCI}^{(23)}$. La elevada frecuencia de estos ítems puede ser justificada por las actividades consideradas de rutina, como la toma de exámenes de laboratorio, que aun así demandan horas de asistencia de enfermería pues las actividades relacionadas a la preparación y administración de medicamentos son parte de los procesos claves al interior de las UCI. La compleja terapia medicamentosa y la administración de medicamentos por vías parenterales son activi- dades frecuentes y complejas y, en ese contexto, el tiempo dedicado a su realización es un elemento crítico, pues los errores de medicamentos son los incidentes más frecuentes que se relacionan con la carga de trabajo de enfermería ${ }^{(2,24)}$.

En cuanto a la baja frecuencia de los ítems referentes a "resucitación cardiopulmonar después de un paro durante las últimas 24 horas", "técnicas de hemofiltración, técnicas de diálisis" y "medición de presión intracraneal", también coincide con otros estudios en donde se han encontrado frecuencias de $0 \%{ }^{(22)}$ para los tres ítems, de $2,1 \%{ }^{(21)}$ y $6,6 \%{ }^{(25)}$ para el ítem "técnicas de hemofiltración, técnicas de diálisis”, de $0,05 \%{ }^{(21)}$ y $0,4^{(25)}$ para el ítem "resucitación cardiopulmonar después de un paro durante las últimas 24 horas", y de $0 \%{ }^{(21,25)}$ para el ítem "Medición de presión intracraneal". Esto puede tener su explicación a que son actividades relacionadas a intervenciones más específicas o con baja frecuencia, que no se aplican a todos los pacientes, como la "medición de presión intracraneal" o la "resucitación cardiopulmonar después de un paro durante las últimas 24 horas".

Respecto de la validez de constructo evaluada con el análisis factorial exploratorio, es necesario destacar que debido a la cantidad de ítems dejados fuera del análisis, por su baja variabilidad, es necesario evaluar el instrumento en una muestra que efectivamente presente las actividades que en esos ítems se encuentran estipuladas, pues quizás el número de pacientes de este estudio influyó en esa baja variabilidad. En contrapartida, respecto de la confiabilidad, aquellos ítems que sí se acoplaron al factor, se pudo observar que funcionaron bien con un estadístico Kuder-Richardson de 0,886, lo que es óptimo. Además, en el análisis de la validez de criterio externo se observó, entre las escalas NAS y TISS-28, una correlación alta, positiva y estadísticamente significativa, resultados concordantes con el estudio de adaptación transcultural y validación del NAS en Brasil ${ }^{(1)}$.

Respecto al análisis factorial exploratorio, cabe señalar que no se encontró en la literatura en el período de realización del presente estudio análisis semejante; solo en España un estudio indicaba que muchos ítems no fueron bien repre- 
sentados y sugieren una estandarización de las puntuaciones $^{(26)}$. Sin embargo, un estudio noruego publicado posterior a la finalización de esta investigación, con el objetivo de probar la confiabilidad entre evaluadores y validez del NAS, realizó un análisis factorial exploratorio y confirmatorio, evidenciando una confiabilidad entre evaluadores significativa y con el apoyo empírico, además, de un buen desempeño del análisis factorial exploratorio y confirmatorio. Los autores sugieren mantener todos los ítems del NAS ${ }^{(27)}$.

En cuanto a las limitaciones de este estudio, la gran cantidad de ítems que no presentaron una comunalidad adecuada, en conjunto con los ítems que quedaron excluidos del análisis por no presentar una variabilidad aceptable, no permitieron ser conclusivos respecto del análisis. En cuanto a la revisión del instrumento original una vez finalizada la adaptación, si bien la literatura recomienda esta etapa ${ }^{(28)}$, la misma no se realiza pues el instrumento ha sido ampliamente utilizado y documentado en su versión original.

\section{CONCLUSIÓN}

No se recomienda la eliminación de ningún ítem del instrumento, debido a la relevancia teórica, práctica y atingencia conceptual de los ítems y se sugiere seguir haciendo estudios de validación en otras poblaciones, con muestras más grandes para favorecer una mayor variabilidad en los puntajes de las actividades que realizan. Se destaca que el instrumento se comportó desde lo esperado, respecto a la evaluación de criterio y consistencia interna, por lo tanto, existe evidencia de validez de criterio externo para el instrumento NAS.

\section{REFERENCIAS}

1. Queijo AF, Padilha KG. Nursing Activities Score (NAS): cross-cultural adaptation and validation to Portuguese language. Rev Esc
Enferm USP. 2009; 43(Esp): 1018-25.

2. Novaretti MC, Santos Ede V, Quitério LM, Daud-Gallotti RM. [Nursing workload and occurrence of incidents and adverse events in ICU patients]. Rev Bras Enferm. 2014; 67(5): 692-9.

3. Berry L, Curry P. Nursing Workload and Patient Care [Internet]. Ottawa: The Canadian Federation of Nurses Unions (CFNU); 2012 [citado 27 jun 2018]. 96 p. Disponible en: https://nursesunions.ca/wp-content/uploads/2017/07/cfnu_workload_printed_version_pdf.pdf

4. Padilha KG, De Sousa RMC, Garcia PC, Bento ST, Finardi EM, Hatarashi RHK. Nursing workload and staff allocation in an intensive care unit: a pilot study according to Nursing Activities Score (NAS). Intensive Crit Care Nurs. 2010; 26(2): 108-13.

5. Miranda DR, Nap R, De Rijk A, Schaufeli W, Iapichino G. The members of the TISS Working Group. Nursing activities score. Crit Care Med. 2003; 31(2): 374-82.

6. Nunes BK, Toma E. Assessment of a neonatal unit nursing staff: application of the Nursing Activities Score. Rev Lat Am Enfermagem. 2013; 21: 348-55.

7. Padilha KG, Stafseth S, Solms D, Hoogendoom M, Monge FJC, Gomaa $\mathrm{OH}$, et al. Nursing Activities Score: an updated guideline for its application in the Intensive Care Unit. Rev Esc Enferm USP. 2015; 49(Spec No): 131-7.

8. Stafseth SK, Solms D, Bredal IS. The characterisation of workloads and nursing staff allocation in intensive care units: a descriptive study using the Nursing Activities Score for the first time in Norway. Intensive Crit Care Nurs. 2011; 27(5): 290-4.

9. Carmona-Monge FJ, Jara-Pérez A, Quirós-Herranz C, Rollán-Rodríguez G, Cerrillo-González I, García-Gómez S, et al. [Assessment of nursing workload in three groups of patients in a Spanish ICU using the Nursing Activities Score Scale]. Rev Esc Enferm USP. 2013; 47(2): 335-40.

10. Sáez E, Infante A. Guías 2004 de Organiza- 
ción y Funcionamiento de Unidades de Pacientes Críticos. Rev Chil Med Inten. 2004; 19(4): 209-223.

11. Alexandre NM, Guirardello E de B. [Cultural adaptation of instruments utilized in occupational health]. Rev Panam Salud Pública. 2002; 11(2): 109-11.

12. De Soárez PC, Kowalski CCG, Ferraz MB, Ciconelli RM. [Translation into Brazilian Portuguese and validation of the Work Limitations Questionnaire]. Rev Panam Salud Pública. 2007; 22(1): 21-8.

13. Lynn MR. Determination and quantification of content validity. Nurs Res. 1986; 35(6): 382-5.

14. Martínez-Arias MR. Psicometría: Teoría de los test psicológicos y educativos. Madrid: Síntesis S.A.; 2005.

15. Fajardo Quintana JC, Cruz Sarmiento M, Mora Y, Torres Leguizamón LM. Validación facial de la escala Nursing Activities Score en tres unidades de cuidado intensivo en Bogotá, Colombia. Enferm glob. 2017; 16(45): 102-15.

16. Keene AR, Cullen DJ. Therapeutic Intervention Scoring System: update 1983. Crit Care Med. 1983; 11(1): 1-3.

17. Lloret S, Ferreres A, Hernándes A, Tomás I. El análisis factorial exploratorio de los ítems: Una guía práctica, revisada y actualizada. Anales de psicología. 2014; 30(3): 1151-1169.

18. Nogueira LS, Ferretti-Rebustini REL, Poveda VB, Silva RCG, Barbosa RL, Oliveira EM, et al. Nursing workload: is it a predictor of healthcare associated infection in intensive care unit? Rev Esc Enferm USP. 2015; 49(Esp): 36-42.

19. Nogueira LS, Sousa RMC de, Padilha KG, Koike KM. Características clínicas e gravidade de pacientes internados em UTIs públicas e privadas. Texto contexto - enferm. 2012; 21(1): 59-67.

20. Altafin JAM, Grion CMC, Tanita MT, Festti J, Cardoso LTQ, Veiga CFF, et al. Nursing Activ- ities Score and workload in the intensive care unit of a university hospital. Rev Bras Ter Intensiva. 2014; 26(3): 292-8.

21. Silva JB, Póvoa VCO, Lima MH, Oliveira HC, Padilha KG, Secoli SR. Nursing workload in hematopoietic stem cell transplantation: a cohort study. Rev Esc Enferm USP. 2015; 49(Spec No): 93-100.

22. Fuly PD, Pires LM, Souza CQ, Oliveira G, Padilha KG. Nursing workload for cancer patients under palliative care. Rev Esc Enferm USP. 2016; 50(5): 792-99.

23. Nogueira LS, Padilha KG, Silva DV, Lança E de F, Oliveira EM, Sousa RM. Pattern of nursing interventions performed on trauma victims according to the Nursing Activities Score. Rev Esc Enferm USP. 2015; 49(Spec No): 29-35.

24. Seynaeve S, Verbrugghe W, Claes B, Vandenplas D, Reyntiens D, Jorens PG. Adverse drug events in intensive care units: a cross-sectional study of prevalence and risk factors. Am J Crit Care. 2011(6); 20: e131-40.

25. Camuci MB, Martins JT, Cardeli AAM, Robazzi MLCC. Nursing Activities Score: nursing work load in a burns Intensive Care Unit. Rev Lat Am Enfermagem. 2014; 22(2): 325-31.

26. Sánchez-Sánchez MM, Arias-Rivera S, Fraile-Gamo MP, Thuissard-Vasallo IJ, Frutos-Vivar F. [Validating the Spanish version of the Nursing Activities Score]. Enferm Intensiva. 2015; 26(2): 63-71.

27. Stafseth SK, Tønnessen TI, Diep LM, Fagerstrom L. Testing the Reliability and Validity of the Nursing Activities Score in Critical Care Nursing. J Nurs Meas. 2018; 26(1): 142162.

28. Chávez LM, Canino G. Toolkit on Translating and Adapting Instruments [Internet]. Human Services Research Institute; 2005 [citado 18 dic 2018]. 58 p. Disponible en: https://www.hsri.org/files/uploads/publications/PN54_Translating_and_Adapting.pdf 